\title{
Diseño de una estación virtual para el control de las perturbaciones que afectan la temperatura de los procesos industriales
}

\author{
Recepción: Febrero de 2007 / Aceptación: Mayo de 2007
}

(1) Jorge Poma Deza (2) Roberto Tello Yuen (3) Edgar Ruiz Lizama

\begin{abstract}
RESUMEN
El artículo presenta el desarrollo de una estación virtual usando LabVIEW, software de instrumentación virtual fabricado por National Instruments, que permite monitorear y supervisar variables en tiempo real en un proceso industrial. Dicho software proporciona una interfaz gráfica de control virtual, toma en cuenta el calor requerido para el proceso en funcionamiento, sin embargo, las interferencias generadas en el proceso logran un incremento de la temperatura del sistema, incorporando una alarma para evitar pérdidas en la producción. Finalmente, se han diseñado opciones de muestreo en el panel de control, como cantidad de datos a medir, tiempo transcurrido, velocidad de muestreo, cantidad de datos tomados, indicar el tiempo en que ha ocurrido algún evento, etc. importantes para facilitar la toma de decisiones.
\end{abstract}

Palabras Clave: Instrumentación virtual, procesos industriales, sistemas de control.

DESIGN OF A VirTUAL CONTROL STATION FOR D IS T URBA NCES THA T AFFECT TEMPERATURE IN INDUSTRIAL PROCESSES

ABSTRACT

The article presents the development of a virtual station using LabVIEW, instrumentation virtual software made by National Instruments, software of virtual instrumentation, which allows monitoring and to supervise variables in real time in an industrial process. This system provides one interface graph of virtual control, considering the heat needed for the process in functioning, nevertheless, the interferences generated towards the process can achieve an increase of the temperature to the system, incorporating an alarm to avoid losses in the production. Finally, options of sampling have been designed in the Control Panel, as quantity of information to measure, passed time, speed of sampling, quantity of taken information, to indicate the time in which some event has happened, etc. Important to facilitate the capture of decisions.

Key Words: Virtual instrumentation, industrial process, control systems.

\section{INTRODUCCIÓN}

En la actualidad, para que las empresas sean competitivas es importante que tomen en cuenta las nuevas tecnologías en procesos industriales a fin de tener un mejor control, toma de decisiones, mayor eficiencia y productividad en todas las áreas de la empresa.

La mejora continua de los procesos debe ser un principio básico de una empresa. En ese sentido el artículo propone una alternativa para optimizar la supervisión y control de la variable temperatura mediante software,monitoreando los efectos producidos por algunas perturbaciones que generan distorsión, interrupción e interferencia, como el sonido, radiaciones, vibraciones, etc., generados por el ambiente, considerándolas a la hora de tomar decisiones adecuadas en la producción.

\section{PROCESOS INDUSTRIALES}

Un proceso es una secuencia de cambios y transformaciones (químico, físico y fisicoquímicos) en la composición de una sustancia utilizando variables como: la temperatura, el flujo, nivel, presión, densidad, volumen, acidez, etc.

Los procesos industriales tienen como propósito principal la transformación de materias primas en un producto final. Durante el proceso de la producción se dividen en procesos como: organización, instalación, operación, formulación, evaluación,simulación, supervisión, etc.; los cuales intervienen en la transformación de los materiales, o convirtiendo energía para producir el producto final.

\section{VARIABLES}

Una variable es una característica que puede tomar diferentes valores en un determinado tiempo. En todo proceso se tiene diversas variables, las cuales afectan a las entradas o salidas del proceso. Temperatura, nivel, flujo y presiónn son las variables más comunes en los procesos industriales, las cuales en la actualidad pueden ser monitoreadas y controladas por medio de la instrumentación Virtual.

\section{Variable de proceso (PV)}

Es el valor de la variable en un instante dado en el proceso. Ejemplo: al

\footnotetext{
Estudiante de Ingeniería Industrial, UNMSM. Personal académico del Centro de Manufactura Avanzada-CEMA, UNMSM. E-mail: jorgeepd@hotmail.com

Ingeniero Industrial. Profesor de1 Departamento de Ingeniería de Sistemas e Informática, UNMSM.

E-mail: rtelloy@unmsm.edu.pe

3) Magíster en Informática. Profesor del Departamento de Ingeniería de Sistemas e Informática, UNMSM

E-mail:eruizl@unmsm.edu.pe
} 
calentar un horno inicialmente está a temperatura ambiente, luego de $5 \mathrm{~min}$, la temperatura está en un valor de $40^{\circ} \mathrm{C}$. para ese instante el valor de $\mathrm{PV}$ es de $40^{\circ} \mathrm{C}$.

\section{Set point (SP)}

Es el valor de consigna o el valor que se desea obtener en el proceso. En el ejemplo anterior el horno está a $40^{\circ} \mathrm{C}$ pero se debe llegar a $70^{\circ} \mathrm{C}$, por lo tanto hay una diferencia de $30^{\circ} \mathrm{C}$, luego el sistema hace que intervenga un actuador (resistencia eléctrica) que permita obtener el valor deseado a $70^{\circ} \mathrm{C}$, para ello existen maneras de controlar los procesos ( $\mathrm{P}, \mathrm{PI}$ , PID, ON OFF, etc.) a fin de obtener el valor deseado y tener el mínimo error.

\section{SISTEMA DE CONTROL}

Es una combinación de elementos conectados entre sí, y que de manera individual no podrían realizar un proceso industrial. Un sistema de control cuenta con 3 elementos:

1. El controlador, es el cerebro del sistema, y por ende proporciona la inteligencia para el sistema de control, como un controlador lógico programable (PLC), una computadora análoga o digital, etc.

2. Los actuadores o transductores de salida, se comportan como los músculos del sistema de control, en el ejemplo anterior, son las resistencias eléctricas que permite aumentar el valor de la variable temperatura, las válvulas, las bombas, etc.

3. Los sensores o transductores de entrada, tienen una función similar a los sentidos del ser humano, miden el valor de las variables relacionados a esas funciones, en un proceso industrial

\section{Sistemas de instrumentación y medición}

Los instrumentos son diseñados para medir, indicar, controlar y almacenar la información de las variables del proceso, además de manipular mecanismos que controlen diversos estados de un proceso. Algunos procesos pueden ser muy similares, pero tienen distintas necesidades de control esto dependerá del nivel de producción.

La medición de una o más variables, hace posible determinar con certeza que sucede en un punto específico del proceso. Un ejemplo de sistema de instrumentación de medición es el sistema de calefacción de una casa es el cual tiene una variable controlada, el termostato de la calefacción indica el estado de la variable existente medida y controlada.

\section{Control de procesos}

El control de procesos consiste en dos funciones claramente diferenciadas: la adquisición de datos y el control; si se trata de establecer el nexo con el mantenimiento se puede afirmar que la adquisición de datos contribuye con la información para el mantenimiento y sus acciones para la implementación.

\section{EL CALOR EN LOS PROCESOS INDUSTRIALES}

\section{Transmisión del calor}

La temperatura es una variable de gran importancia, pues en la industria existen procesos físicos y químicos que solo proceden en un rango determinado de temperatura, por esta razón, es importante el control apropiado de esta variable.

Los mecanismos básicos de transmisión de calor son: conducción, convección y radiación, no solo trabajan independientemente sino que en la mayoría de los casos el calor se transfiere simultáneamente en más de una forma.

Las leyes de transmisión del calor son de suma importancia en el diseño y funcionamiento de múltiples equipos como hornos, generadores de vapor, precalentadores, intercambiadores de calor, recuperadores de calor y regeneradores de calor, etc.

\section{Modelos matemáticos de la ecuación del calor}

La ecuación del calor es un modelo matemático expresado mediante una ecuación diferencial, que trata de describir la evolución de la temperatura en un cuerpo sólido.

Por ejemplo, una barra metálica aislada de longitud uno, inicialmente a temperatura cero que después de un cierto tiempo $t_{0}$ se calienta a una temperatura $\mathrm{T}\left(x, t_{0}\right)$ manteniendo sus extremos $\mathrm{x}=0$ y $\mathrm{x}=1$ a temperatura cero, a partir de ese instante $t_{0}$ se deja que la temperatura $\mathrm{T}\left(x, t_{0}\right)$ evolucione libremente y se necesita un modelo matemático que permita predecir la temperatura $\mathrm{T}\left(x, t_{0}\right)$ para todo $\mathrm{x}$ en el intervalo $[0,1]$, en todo tiempo futuro, $t>t_{0}$ y para $\mathrm{x}=0 \quad \mathrm{O} \quad \mathrm{x}=1$.

Se toma un trozo de barra, la energía contenida en $\square l$ en el instante $t_{2}$ es igual a la energía que había en $\square l$ en el instante $t_{1}$ más el "flujo de energía" que penetró a los extremos $X_{1}, X_{2}$ en el intervalo de tiempo de $t_{1}$ a $t_{2}$, matemáticamente, su modelo es el siguiente:

$$
\int_{\square l}\left(e\left(x, t_{2}\right)-(e(x, t 1)) d x=\int_{t_{1}}^{t_{2}}\left(-f\left(x_{2}, t\right)+f(x 1, t)\right) d t\right.
$$


Existen infinitos modelos, dependiendo de la precisión y el rango de valores válidos (altas o bajas temperaturas) que cambiarán el comportamiento del material, impurezas podrían ser relevantes, etc.

\section{MANERAS DE CONTROLAR LOS PROCESOS}

OnOff: Es el más sencillo de todos, ya que la señal sólo tiene dos estados posibles. Así, en el caso de una válvula, o está totalmente abierta o totalmente cerrada.

Proporcional $(\mathbf{P})$ : Es un tipo de control que varia de manera proporcional sea directamente o inversamente proporcional en función del error en el proceso y la interviene del actuador en el proceso.

$$
\mathrm{c}(\mathrm{t})=\mathrm{K}_{\mathrm{c}} \Gamma_{(\mathrm{t})}+\mathrm{c}_{\mathrm{s}}
$$

Donde: $c(t)$ : señal a un tiempot.

$$
\text { Kc : ganancia proporcional del }
$$

$\hat{i}(\mathrm{t}) \quad$ : error a un tiempo $\mathrm{t}$.

cs : señal que sale del controlador cuando el error es cero.

Proporcional-integral (PI): Su señal de actuación es la misma que la del controlador proporcional, a la cual se le añade un término integral. También se conoce como controlador proporcional + reset.

$$
\mathrm{c}(\mathrm{t})=\mathrm{K}_{\mathrm{c}} \Gamma(\mathrm{t})+\frac{\mathrm{K}_{\mathrm{c}}}{\mathbf{I}_{\mathrm{I}}} \square_{0}^{\mathrm{t}} \Gamma(\mathrm{t}) \mathrm{dt}+\mathrm{c}_{\mathrm{s}}
$$

Donde $\mathrm{T}$, representa la constante de tiempo integral o tiempo de reset (tiempo que necesita el controlador para repetir la acción inicial de control proporcional en su salida).

\section{Proporcional-integral-derivativo (PID):}

Es igual que un controlador proporcional más un término integral y otro término derivativo. La señal de actuación viene dada por:

$$
\mathrm{c}(\mathrm{t})=\mathrm{K}_{\mathrm{c}} \Gamma(\mathrm{t})+\frac{\mathrm{K}_{\mathrm{c}}}{\mathrm{A}_{\mathrm{I}}} \zeta_{0}^{\mathrm{t}} \Gamma(\mathrm{t}) \mathrm{dt}+\mathrm{K}_{\mathrm{c}} \boldsymbol{\mathrm { D }}_{\mathrm{D}} \frac{\mathrm{d} \Gamma}{\mathrm{dt}}+\mathrm{c}_{\mathrm{s}}
$$

Ahora, ${ }_{D}$ representa la constante de tiempo.

\section{PERTURBACIONES}

Son agentes que alteran el desarrollo de un proceso industrial, actuando de diferentes maneras, siendo los modelos matemáticos más frecuentes son las de tipo escalon, rampa, senoidal, pulso, impulso y exponencial.

\section{INSTRUMENTACIÓN VIRTUAL}

El concepto de instrumentación virtual nace a partir del uso del computador personal (PC) como "instrumento" de medición de tales señales como temperatura, presión, caudal, etc.

Es decir, el PC apoyado de hardware para la adquisición de datos comienza a ser utilizado para realizar mediciones de fenómenos físicos representados en señales de corriente (Ej. 4-20mA) y/o voltaje (Ej. (0-5Vdc). Sin embargo, el concepto de "instrumentación virtual" va más allá de la simple medición de corriente o voltaje, sino que también involucra el procesamiento, análisis, almacenamiento, distribución y despliegue de los datos e información relacionados con la medición de una o varias señales específicas. Es decir, el instrumento virtual no se conforma con la adquisición de la señal, sino que también involucra la interfaz hombremáquina, las funciones de análisis y procesamiento de señales, las rutinas de almacenamiento de datos y la comunicación con otros equipos.

\section{Instrumentación Virtual con LabVIEW}

LabVIEW es hoy una plataforma estándar en la industria de test y medida para el desarrollo de sistemas de prueba y control de instrumentación, en el campo de la automatización industrial para la adquisición de datos, análisis, monitorización y registro, así como para el control y monitorización de procesos, en el área de visión artificial para el desarrollo de sistemas de inspección en producción o laboratorio.

\section{Adquisición de datos}

La adquisición de datos es el proceso que involucra la recopilación de información de una forma automatizada a partir de fuentes de medición análogas y digitales como sensores.

En la adquisición de datos se utiliza una combinación de hardware de medición y software con apoyo en una PC para proporcionar un sistema de medición flexible y definido por el usuario.

\section{DISEÑO E IMPLEMENTACIÓN DE LA ESTACIÓN DE CONTROL}

Consiste en la realización de la interface para visualizar y monitorear las variables del proceso. Dicha interface se realiza en el Front Panel de la pantalla del LabVIEW.

En dicho panel se define los controladores y las variables del programa, representando el visualizador gráfico, sistema de botonería, alarma, 
Figura 1. Esquema del proceso

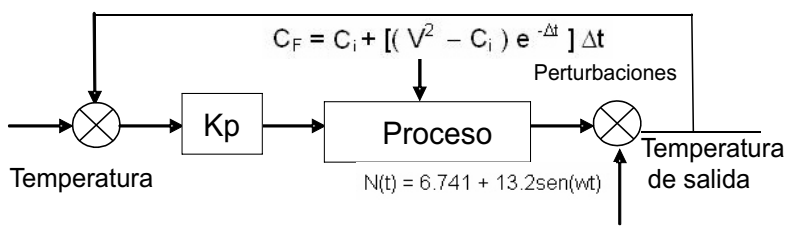

Fuente: Elaboración propia (2006)

luces, instrumentación, etc. de la estación virtual, de la siguiente manera:

Dispositivos de entrada, la cual recibe las variables físicas a medir, enviando una señal un acondicionador de señal o de procesamiento y un dispositivo de salida. El dispositivo de entrada recibe la variable física a medir y envía una señal eléctrica proporcional al dispositivo de salida, aquí se amplifica, se filtra o se modifica en un formato adecuado, y van a los dispositivos de salida, los cuales pueden ser, una computadora digital para la manipulación de los datos (como en este caso), o un Interface Hombre-Máquina (HMI, por sus siglas en inglés).

\section{Diseño del sistema de control}

En la figura 1 se puede apreciar el diseño de un sistema de Control Proporcional, con el fin de obtener mayor precisión en el desarrollo de la aplicación. Aquí se puede apreciar una temperatura entrante al sistema, que pasa por un proceso, el cual es regido por un modelo matemático, el cual se ve interrumpido por distorsiones. Para hacer más entendible y didáctica la explicación, a lo largo del proyecto estoy tomando valores reales captados en un proceso industrial mediante tarjetas de adquisición de datos.

\section{Diseño del panel de monitoreo de variables físicas}

Las señales vinculadas de los sensores, actuadores, controladores y tarjetas de adquisición de datos son monitoreadas en el Front Panel del LabVIEW.

Se puede encontrar en dicho panel, los siguientes elementos:

1. Visualizador gráfico: En dicha pantalla se muestra la variación de las señales, con respecto al tiempo.

a. La primera señal (color rojo) corresponde al valor de la temperatura inicial.

b. La segunda señal (color verde) corresponde al valor real de la temperatura usada en el proceso, es decir, luego que ha sido afectada por las perturbaciones.
2. Sistema de alarmas: Que permitirá tomar decisiones adecuadas según el valor de la temperatura.

a. La alarma de límite máximo, me permite controlar el proceso para que no hayan pérdidas en la producción, ni en el sistema debido a un sobrecalentamiento en el proceso.

b. La alarma de rango, permite determinar si el proceso está en un par de puntos definidos según el tipo de proceso o producto.

3. Ingreso de temperatura: Definido mediante un knob o mediante teclado, el cual puede ser visualizado con el termómetro gráfico del sistema.

4. Velocidad de muestreo: Controla la velocidad de visualización de las gráficas, mientras sea más lenta, habrá mayor facilidad de análisis gráfico.

5. Kp, es la constante proporcional de control del sistema.

6. Controlador de tiempo, muestra visualmente el tiempo en segundos que está transcurriendo desde iniciado el proceso, cuenta con una opción para ver el tiempo en que ha sido tomada cierta muestra, así como para reiniciar el conteo en el proceso.

En la figura 2 se aprecia el panel de monitoreo, para el modelo del esquema del proceso.

\section{Estrategia de programación del sistema}

Se realiza en el Block Diagram del LabVIEW, aquí se realizan todas las estrategias de programación, que se pude apreciar en la figura 3 .

1. Visualizador gráfico: Representado mediante un Waveform Graph, el cual recibe dos señales unidas mediante un Unbundle.

a. La primera señal (color rojo) corresponde al valor de la temperatura inicial del proceso, el cual también se puede visualizar en el panel.

b. La segunda señal (color verde) corresponde al valor real de la temperatura la cual ha pasado por la ecuación o modelo matemático de las perturbaciones.

2. Sistema de alarmas: Consiste en comparar valores de la temperatura en determinados rangos, los cuales son indicados mediante leds.

a. La alarma de límite máximo, consiste en comparar el valor de ingreso de temperatura al proceso, con un valor fijado por el usuario, dependiendo del producto, o en su defecto se 
Figura 2. Panel frontal del proceso

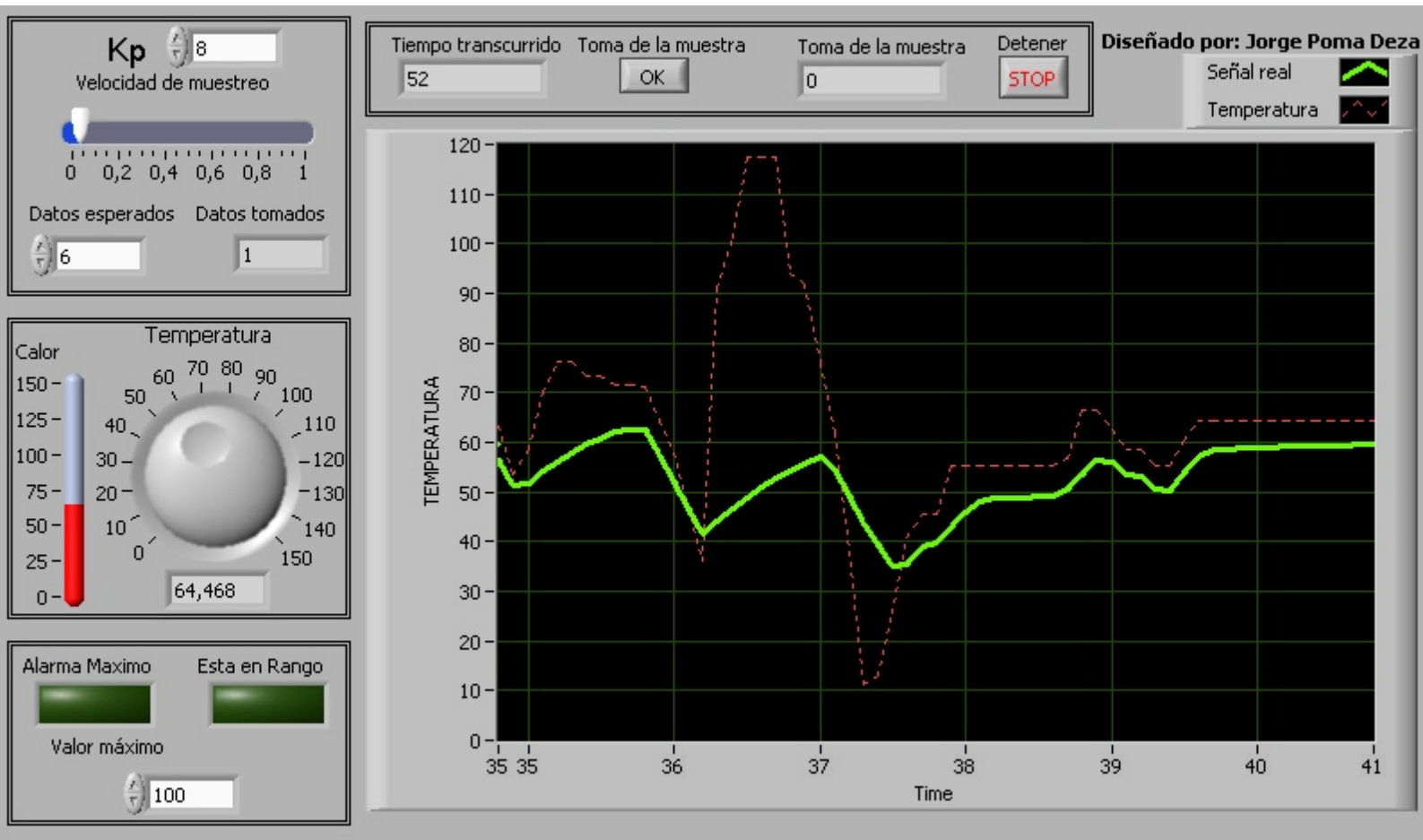

Fuente: Elaboracion propia (2006)

Figura 3. Diagrama de bloques del proceso

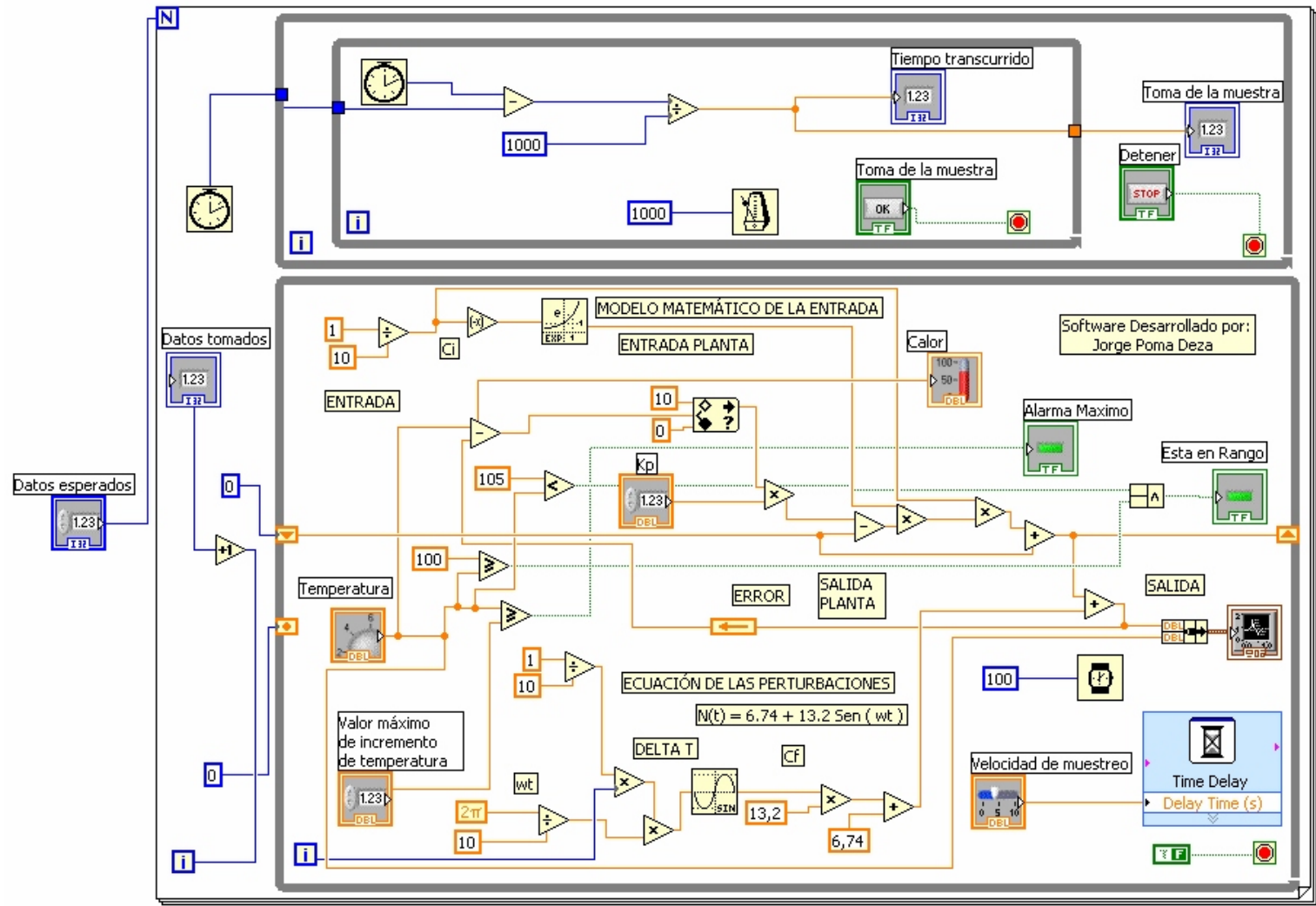

Fuente: Elaboración propia (2006) 
poner el valor máximo de temperatura permitido por los dispositivos del sistema, para evitar cualquier incidente no previsto en la planta.

b. La alarma de rango, me permite controlar el calor necesario según el tipo de producto, para nuestro proceso, se necesita un rango entre los 100 y $105^{\circ} \mathrm{C}$.

3. Ingreso de temperatura: Se puede controlar ese ingreso mediante PC al proceso, así como el aumento y disminución de manera automática, mediante un Controlador y visualizado en el termómetro, el cual recibe ese valor $\mathrm{y}$ es visualizado en el tablero.

4. Velocidad de muestreo: Mediante un time delay conectado a un controlador, para poder variar la velocidad de muestreo, y ver las gráficas según el análisis requerido.

5. Controlador de tiempo, funciona como un cronómetro, se tiene un tiempo en este caso lo hemos fijado cada un segundo, tiene una opción para detener el tiempo, una para guardar el último valor almacenado y el final, para reiniciar el proceso.

\section{Etapa de acondicionamiento de señales}

Para recolectar la información generada por todos los instrumentos y dispositivos, y el manejo de entrada y salida de datos y de proceso, se cuenta con la etapa de adquisición de datos.

La tarjeta de adquisición de datos empleada es la USB-6008 de National Instrument, además de dispositivos de entrada/salida, entradas analógicas, entradas digitales, salidas digitales, contadores y relojes, PLC's.

Figura 4. Acondicionador cambiador de escala

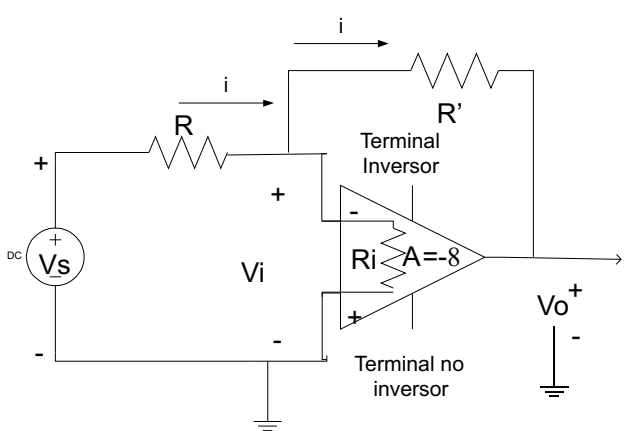

Fuente: Elaboración propia (2007)

En esta etapa se realizan diversas operaciones para adecuar las señales (analógicas y digitales) del proceso a los parámetros que necesitan la tarjeta de adquisición de datos USB-6008 de Nacional Instruments. Para los elementos utilizados en esta aplicación se necesitan cuatro etapas de acondicionamiento.

\section{Acondicionador Cambiador de Escala}

El cual cumple una función amplificadora entre la entrada y salida, por ejemplo al tener una entrada de $\pm 100 \mathrm{mV}$, pero la tarjeta USB-6008 una entrada de $\pm 10 \mathrm{~V}$, para lograr dicho efecto, se usa el Amplificador Operacional Realimentado Inversor como se muestra en la figura 4.

De la figura 5, la ganancia de tensión Av es:

$$
A v=\frac{V_{o}}{V_{s}}=\frac{-R^{\prime}}{R}=\frac{-Z^{\prime}}{Z}
$$

Si se define el factor de cambio de escala como "k", puesto que se tiene un voltaje de entrada y uno salida, dicho factor es equivalente a la relación $\mathrm{R}^{\prime} / \mathrm{R}$, por lo tanto:

$$
A v=-k=\frac{V_{\mathrm{o}}}{V_{\mathrm{s}}}
$$

En el circuito equivalente que se muestra en la figura 5 se observa que una excursión de entrada de $\pm 100 \mathrm{mV}$ es en realidad una excursión total de $200 \mathrm{mV}$, como la tarjeta de adquisición de datos requiere $10 \mathrm{~V}$, en realidad necesita $20 \mathrm{~V}$, lo cual se muestra en la siguiente relación:

$$
-\mathrm{k}=\frac{\mathrm{V}_{\mathrm{o}}}{\mathrm{V}_{\mathrm{s}}}=\frac{20 \mathrm{~V}}{200 \mathrm{mV}}=\frac{20 \mathrm{~V}}{0.2 \mathrm{~V}}=100
$$

Figura 5. Circuito equivalente del acondicionador cambiador de escala

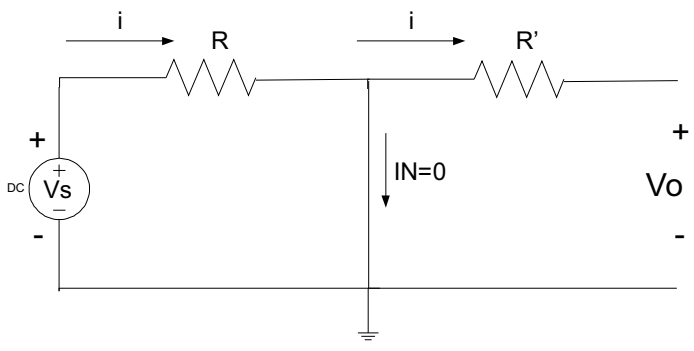

Fuente: Elaboración propia (2007) 
Figura 6. Acondicionador cambiador escala.

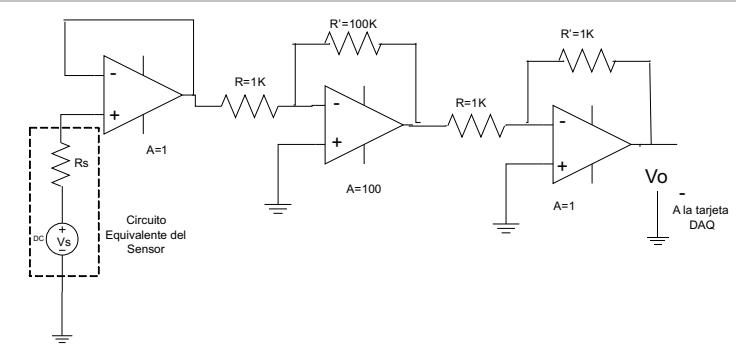

Fuente: Elaboración propia (2007)

Como la relación de resistencias es de 100 a 1 , se necesita una resistencia de $1 \mathrm{~K} \Omega$ para la resistencia de $100 \mathrm{~K} \Omega$. En la figura 6 se visualiza el diseño final del acondicionador para la aplicación.

\section{Acondicionador Integrador}

Para la conversión frecuencia-tensión de los controladores de muestreo.

Aprovechando la estrategia de diseño anterior, se propone reemplazar $\mathrm{R}^{\prime}$ por un condensador $\mathrm{C}$, al circuito anterior y se obtiene el circuito de la figura 7.

Se demuestra que este circuito realiza la operación de integración, para ello se elabora su circuito equivalente, el cual se muestra en la figura 6.

Se tiene una entrada que opera en función del tiempo $v=v(t)$, que en conjunto con la carga del condensador $\mathrm{C}$, generan una $\mathrm{i}=\mathrm{i}(\mathrm{t})$, en la figura 8 se aprecia que la unión de la resistencia $R$ y el condensador están al mismo voltaje que la conexión a tierra, por ende, ambos puntos representan un "cortocircuito virtual".

Se sabe que $V_{o}=\frac{1}{C} \square$ i. $d t$

Figura 8: Circuito equivalente del Integrador Operacional

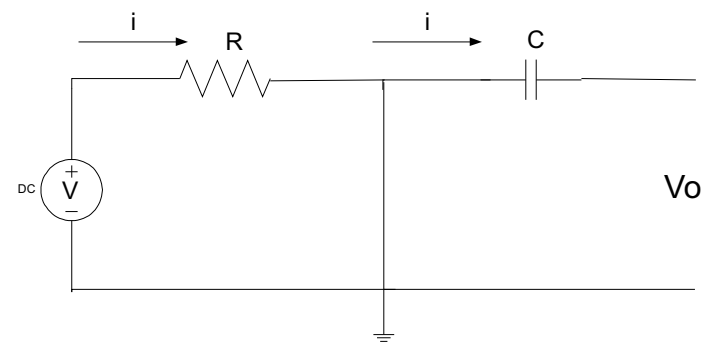

Fuente: Elaboración propia (2007)
Figura 7. Acondicionador Integrador

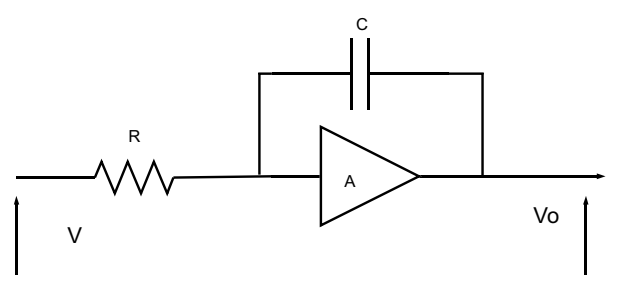

Fuente: Elaboración propia (2007)

Se aplica la Ley de Ohm en la figura 8, y se obtiene:

$$
V_{o}=-\frac{1}{R C} \square \mathrm{v} \cdot d t
$$

Se observa que la tensión de salida Vo ha resultado igual a la integral de la tensión de entrada.

Para la aplicación el valor de v es constante e igual a $\mathrm{V}$, por lo que la integral queda:

$$
V_{o}=-\frac{V . t}{R C}
$$

Como la señal está formada por pulsos cuya frecuencia se quiere medir y son ingresados al sistema integrador, a medida que transcurre el tiempo se sucederán más pulsos y la salida será función de la cantidad de pulsos que ingresaron, se deduce que el integrador funcionará como contador.

\section{Acondicionador Convertidor de Tensión a Corriente}

Usado para enviar señales o data a través de la tarjeta de adquisición de datos hacia en los diversos dispositivos del sistema, por ejemplo, para accionar la resistencia eléctrica que eleva la

Figura 9. Amplificador Operacional Inversor con retroalimentación de tensión en paralelo

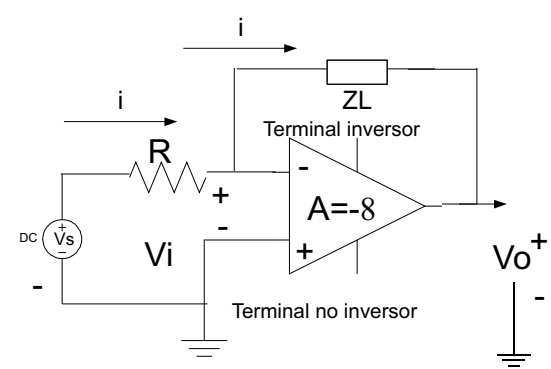

Fuente: Elaboración propia (2007) 
Figura 10: Tierra virtual en el Amplificador Operacional

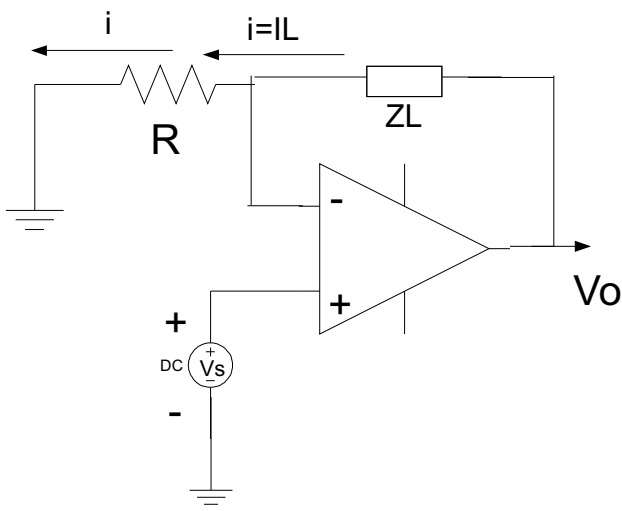

Fuente: Elaboración propia (2007)

En las figuras 9 y 10 se realizan una modificación al diseño inicial, cambiando las resistencias R' por la entrada del equipo en el cual se intenta dar una orden, que será recibida en forma de corriente. Asimismo, se simboliza como carga ZL al actuador que se reemplaza por R'.

De esa manera, se obtiene un convertidor de tensión a corriente porque la corriente i es la misma que circula por ZL, cuyo valor de corriente es:

$$
I L=\frac{V s(t)}{R}
$$

Al ser Vs directamente proporcional con IL, ambas varian en función del tiempo según $\mathrm{Vs}(\mathrm{t})$, debido a que en la conexión del amplificador operacional retroalimentado inversor, la entrada inversora está al mismo potencial que la tierra aunque eléctricamente no esté conectada a ella (tierra virtual), obteniendo el siguiente diseño final:

Figura 11: Convertidor de Tensión a corriente

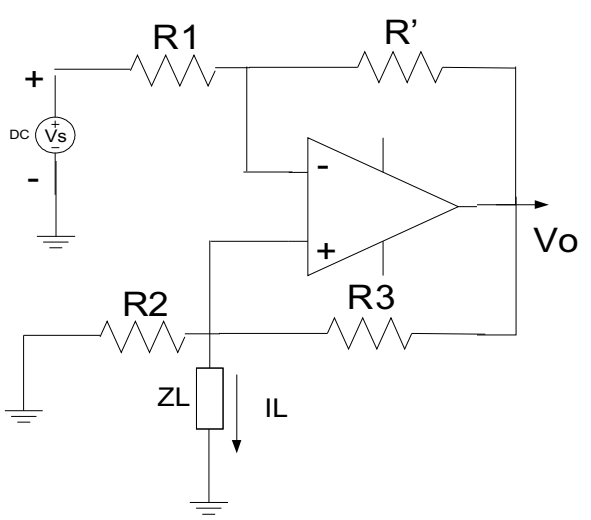

Fuente: Elaboración propia (2007)
4. Acondicionador Convertidor de Corriente a Tensión, usado para los sensores cuya función es la toma de datos con salidas de corriente, mientras que la tarjeta de adquisición de datos tiene entradas de tensión, por ello es necesario colocar un acondicionador de señal que convierta la corriente del sensor en una tensión para que se pueda ingresar a la placa DAQ.

Debido a la tierra virtual a la entrada del inversor del amplificador, la corriente en la resistencia Rs es cero e Is, circula totalmente por la resistencia R'. Por lo tanto, la tensión Vo es igual pero de signo contrario a la caída de potencial, que la corriente Is que se produce en la resistencia R'. Entonces:

$$
V o=-I s \cdot R^{\prime}
$$

Se propone la figura 12, cuya entrada es is es la salida del sensor, siendo Vo el resultado final, que sería la entrada de tensión a la DAQ.

Finalmente, se agrega un condensador $C^{\prime}$ que tiene la función de filtrar los diversos ruidos y perturbaciones de alta frecuencia que se pueden originar en el acondicionador.

\section{CONCLUSIONES}

Es importante considerar en los procesos industriales adicionalmente al "control de procesos", las herramientas de software de interfaz gráfica para controlar y supervisar dichos procesos, a fin de mejorar la calidad del producto.

Se puede simular el control de agentes externos que modifican las condiciones estables de un proceso industria, para minimizar el efecto distorcionante

Figura 12: Convertidor de corriente a tensión

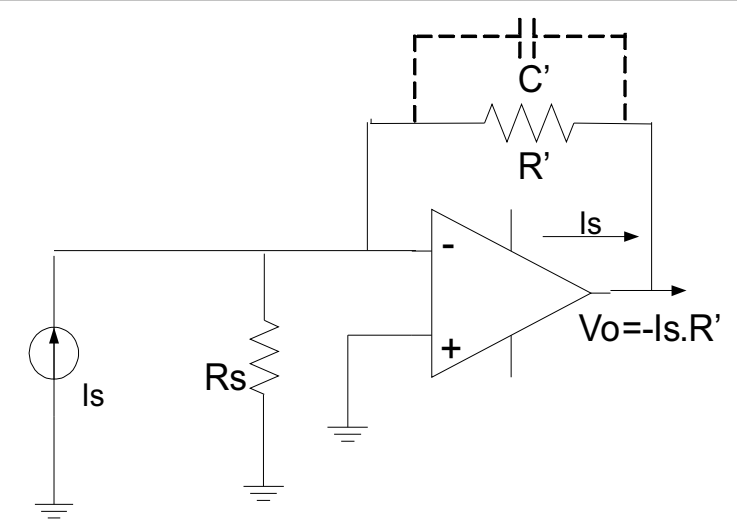

Fuente: Elaboración propia (2007) 
logrando sintonizar adecuadamente dichas variables según especificaciones técnicas de producción.

LabVIEW de National Instruments es una herramienta de software de interfaz gráfica muy poderosa, flexible y amigable que nos permite representar esquemáticamente en la pantalla de un computador a través de un $\mathrm{HMl}$ las variables que son leídas de un controlador (PLC) que a su vez está comunicado con los instrumentos de campo quienes toman la información de la variable a controlar como, por ejemplo, la temperatura.

Es importante complementar el trabajo de un "instrumento virtual" desarrollado en LabVIEW para almacenar las variables controladas en una base de datos externa como SQL Server, Oracle, Access u otro; para que luego con esta información se pueda mejorar y apoyar al planeamiento y control de la producción, mantenimientos preventivos, correctivos y soporte a los ERP (Planeamiento de Recursos Empresariales).

\section{REFERENCIAS BIBLIOGRÁFICAS}

1. Acedo, J. (2002). Control Avanzado de Procesos, Edit. Diaz de Santos, Madrid, España.

2. Arbildo, A. y Calderón, E. (2005). Manual del Módulo de Control de Temperatura, Sociedad Inducontrol Ingeniería S.A.C., Lima, Perú.
3. Caffarelli Luis (2003). Centro Virtual de divulgación de las matemáticas. En: http://www.divulgamat.net/weborriak/TestuakOnLi ne/Hasierakolkasgaiak/caffarelli2003-04.pdf (Visitado:27-11-06).

4. Calderón, E. y Vinces, N. (2006). Curso Taller: LabVIEW: Software para el Control y Automatización de Procesos industriales, Centro de Manufactura Avanzada, Facultad de Ingeniería Industrial, Universidad Nacional Mayor de San Marcos, Lima, Perú.

5. National Instruments. (2005). LabVIEW: User Manual, National Instruments Corporation, Austin, United Status. En: www.ni.com/pdf/manuals/320999e.pdf (Visitado:27-11-06).

6. Poma, J. Félix, G. Tello, R. (2006). Manual del curso de Laboratorio de Operaciones y Procesos Unitarios, Centro de Manufactura Avanzada, Facultad de Ingeniería Industrial, Universidad Nacional Mayor de San Marcos, Lima, Perú.

7. Rosero, W. Gutiérrez, W. y Mantilla, W. (2006). Curso Internacional de Supervisión y Automatización totalmente integrada, Centro de Electricidad y Automatización Industrial (CEAI), Servicio Nacional de Aprendizaje (SENA), Cali, Colombia. 